\title{
The CERN Large Hadron Collider as a Tool to Study High-Energy Density Matter
}

\author{
N. A. Tahir, ${ }^{1}$ V. Kain, ${ }^{2}$ R. Schmidt, ${ }^{2}$ A. Shutov, ${ }^{3}$ I. V. Lomonosov, ${ }^{3}$ V. Gryaznov, ${ }^{3}$ A. R. Piriz, ${ }^{4}$ M. Temporal, ${ }^{4}$ \\ D. H. H. Hoffmann, ${ }^{1,5}$ and V. E. Fortov ${ }^{3}$ \\ ${ }^{1}$ Gesellschaft für Schwerionenforschung, Planckstraße 1, 64291 Darmstadt, Germany \\ ${ }^{2}$ CERN-AB, 1211 Geneva 23, Switzerland \\ ${ }^{3}$ Institute of Problems of Chemical Physics, Russian Academy of Sciences, Institutskii pr. 18, 142432 Chernogolovka, Russia \\ ${ }^{4}$ E.T.S.I.Industriales, Universidad de Castilla-La Mancha, 13071 Ciudad Real, Spain \\ ${ }^{5}$ Institut für Kernphysik, Technische Universität Darmst adt, Schlossgartenstraße 9, 64289 Darmstadt, Germany
} (Received 17 August 2004; published 8 April 2005)

The Large Hadron Collider (LHC) at CERN will generate two extremely powerful $7 \mathrm{TeV}$ proton beams. Each beam will consist of 2808 bunches with an intensity per bunch of $1.15 \times 10^{11}$ protons so that the total number of protons in one beam will be about $3 \times 10^{14}$ and the total energy will be $362 \mathrm{MJ}$. Each bunch will have a duration of $0.5 \mathrm{~ns}$ and two successive bunches will be separated by $25 \mathrm{~ns}$, while the power distribution in the radial direction will be Gaussian with a standard deviation, $\sigma=0.2 \mathrm{~mm}$. The total duration of the beam will be about $89 \mu \mathrm{s}$. Using a 2D hydrodynamic code, we have carried out numerical simulations of the thermodynamic and hydrodynamic response of a solid copper target that is irradiated with one of the LHC beams. These calculations show that only the first few hundred proton bunches will deposit a high specific energy of $400 \mathrm{~kJ} / \mathrm{g}$ that will induce exotic states of high energy density in matter.

DOI: $10.1103 /$ PhysRevLett.94.135004

PACS numbers: 52.27.Gr, 52.50.Lp

The motivation to construct the Large Hadron Collider (LHC) at CERN comes from fundamental problems in Particle Physics. The LHC is being installed in a tunnel with a circumference of $26.8 \mathrm{~km}$ that was previously used for the Large Electron Positron Collider (LEP). Two counter rotating proton beams will be made to circulate in separate beam pipes and will be accelerated to particle energies of $7 \mathrm{TeV}$. The protons in the two beams will then be made to collide at the center of mass energy of $14 \mathrm{TeV}$.

Each beam will consist of a bunch train with every bunch consisting of $1.15 \times 10^{11}$ protons. The total number of bunches will be 2808 , so that the total number of protons in each beam will be $3 \times 10^{14}$. The bunch length will be $0.5 \mathrm{~ns}$ and two neighboring bunches will be separated by $25 \mathrm{~ns}$ while the radial power profile in the beam spot will be Gaussian with a standard deviation of $0.2 \mathrm{~mm}$. The total duration of the beam is of the order of $89 \mu \mathrm{s}$.

It is interesting to note that a very important "spinoff" of the LHC could be the studies of high-energy density (HED) states in matter, because interaction of such powerful beams with solid matter would lead to creation of these exotic states. These include expanded as well as compressed hot liquid states, two phase liquid-gas region, critical point region, and strongly coupled plasma states including exotic phase transitions in nonideal plasmas. Study of fundamental properties of these exotic states of matter, especially the equation of state (EOS) properties, is of considerable interest to many branches of basic and applied physics. For example, astrophysics, planetary sciences, inertial fusion, and many others.

Standard techniques of creating HED matter involve shock compression of matter using different shock wave generators. Pressures in the 10 Mbar range have been produced employing high power explosives, light gas guns and $Z$ pinches [1-5]. Much higher pressures in the Gbar range have been generated using underground nuclear explosions [6] and powerful lasers [7-10]. Recently it has been proposed to employ intense ion beams to induce HED states in matter using isochoric heating without generation of shock waves [11]. This latter method has a number of advantages over the traditional methods of using shock waves. Like an intense heavy ion beam, the LHC beam will also be a very powerful tool to research this interesting field of physics.

In order to study the potential of using the LHC beams for this purpose, we carried out numerical simulations of the thermodynamic and hydrodynamic response of a solid copper target that is irradiated with one of the LHC beams. We note that for each of the two LHC beams there is a beam dumping system that extracts the beam after normal operation as well as in the case of equipment failure. The extracted beam is transferred through a $700 \mathrm{~m}$ long beam line to the beam dump block. The extraction is fast, all bunches are extracted within one turn. The beam dumping system is one of the important safety measures required during the LHC operation. In principle, it would allow extracting a $7 \mathrm{TeV}$ beam onto a copper target and this could be done safely. However, this may require some modifications of the beam extraction line. The simulations have been carried out using a two-dimensional computer code BIG-2 [12] which is based on a Godunov type numerical scheme. A multiphase semiempirical EOS model [13] is used to treat different phases of the target material. Our simulations show that the first bunch deposits about 


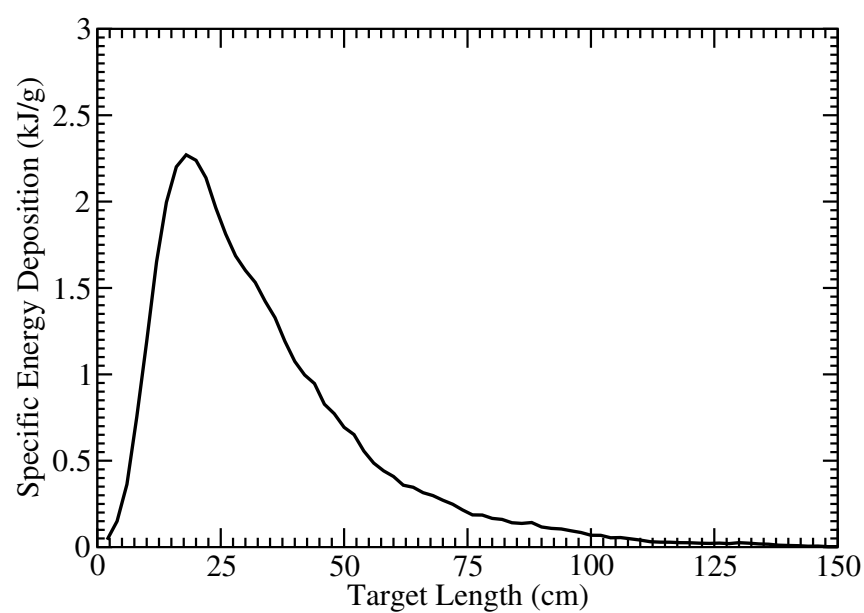

FIG. 1. Specific energy deposited by one bunch along the target axis (time $=1 \mathrm{~ns}$ ).

$2.5 \mathrm{~kJ} / \mathrm{g}$ specific energy in the target. The specific energy deposition increases as the subsequent bunches deliver their energy to the target.

It is well known that energetic heavy ions deposit their energy in the target as a result of Coulomb collisions, mainly with the target electrons $[14,15]$. The $7 \mathrm{TeV}$ protons, on the other hand, when incident on matter, will generate particle cascades in all directions and one needs to calculate the energy deposited by all these different particles in the target. For this purpose we have used the well known particle interaction and transport Monte Carlo code, FLUKA [16]. This code is capable of calculating all components of particle cascades in matter from $\mathrm{TeV}$ energies down to that of the thermal neutrons. The energy deposition profile calculated by the FLUKA code is used as input to the BIG-2 code. The target geometry for the FLUKA simulations is considered to be a cylinder of solid copper that is $5 \mathrm{~m}$ long and has $1 \mathrm{~m}$ radius. The energy deposition

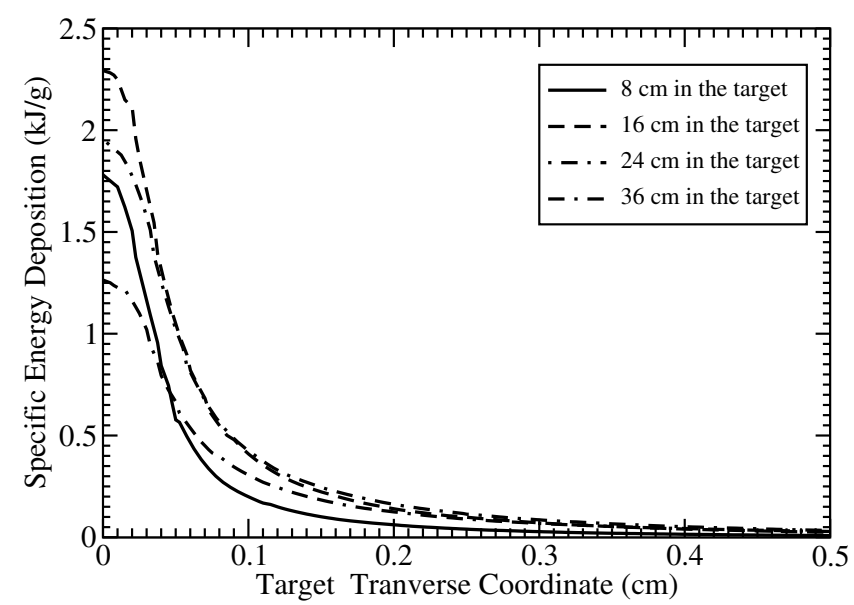

FIG. 2. Specific energy vs transverse coordinate at different longitudinal positions (time $=1 \mathrm{~ns}$ ).

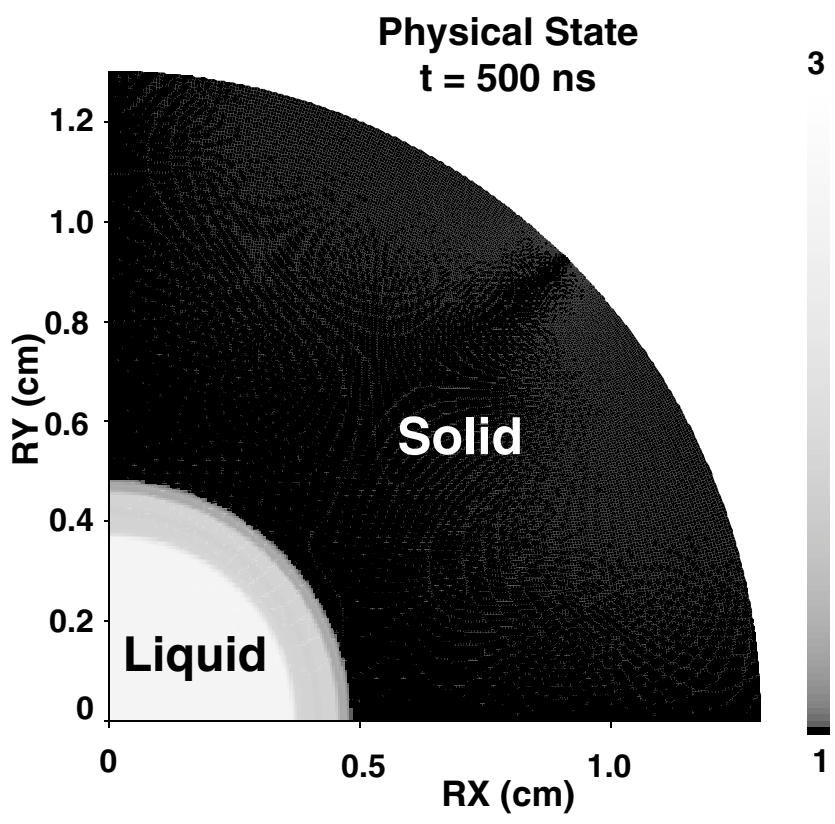

FIG. 3. Material state at $t=500 \mathrm{~ns}$.

profile along the target axis per bunch (after $1 \mathrm{~ns}$ ) is shown in Fig. 1.

The longitudinal peak of energy deposition occurs at about $15 \mathrm{~cm}$ and the deposited energy is a factor 1250 lower than the peak value at about $1.5 \mathrm{~m}$. Energy deposition profiles along the transverse direction at four different points along the length, namely, $8,16,24$, and $36 \mathrm{~cm}$ are plotted in Fig. 2.

For the BIG-2 calculations we consider a cylinder made of solid copper with a radius of $5 \mathrm{~cm}$ and the beam is

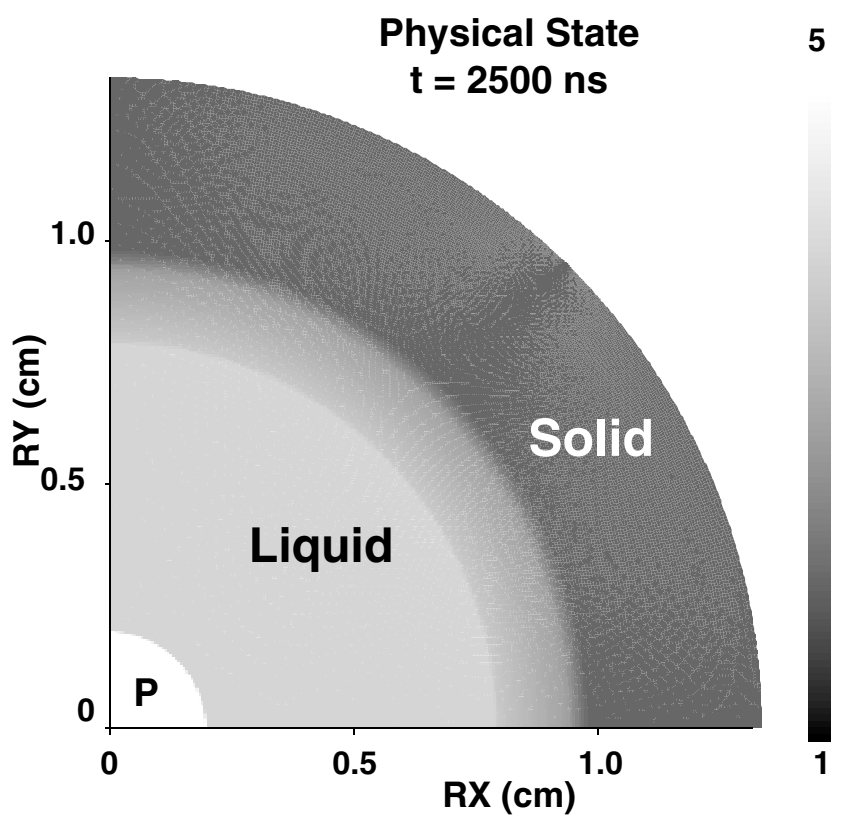

FIG. 4. Material state at $t=2.5 \mu \mathrm{s}$. 


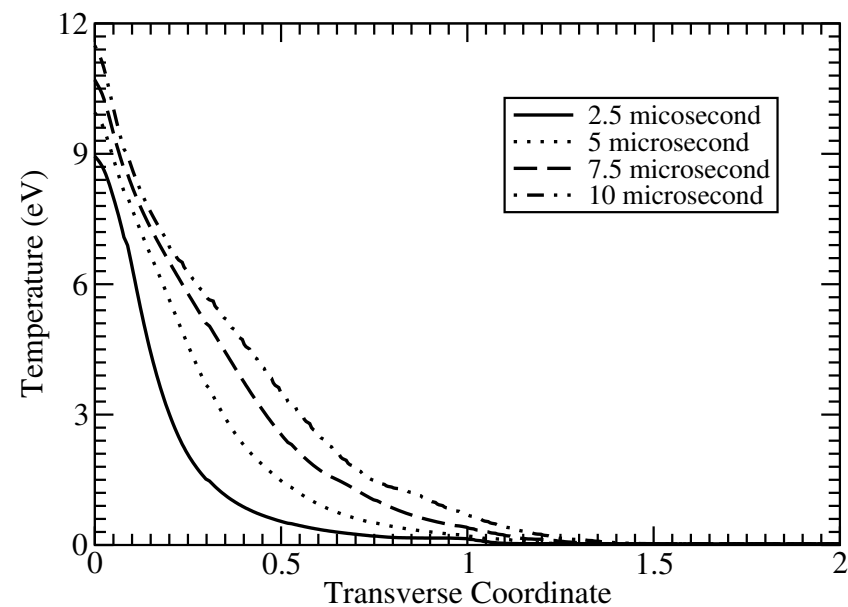

FIG. 5. Temperature vs transverse coordinate at different times at a longitudinal position of $16 \mathrm{~cm}$.

incident along the axis. Because of the three-dimensional nature of the energy deposition calculated by the FLUKA code, and the limitation that the BIG-2 code is twodimensional, we simulate target heating and expansion in the transverse plane at different longitudinal positions, $L$. Here we only report results corresponding to $L=16 \mathrm{~cm}$ where the maxima of the energy deposition lies.

In Fig. 3 we present the material state along the transverse plane of the target at $L=16 \mathrm{~cm}$ and at $t=500 \mathrm{~ns}$. By this time a specific energy of $44 \mathrm{~kJ} / \mathrm{g}$ has been deposited in the target that leads to a temperature of $5 \mathrm{eV}$ and a pressure of $35 \mathrm{GPa}$. The high pressure drives a radially outgoing shock wave and as a consequence the density at the target center decreases to a value of $3.6 \mathrm{~g} / \mathrm{cm}^{3}$. It is seen that the material that lies within a radius of $4 \mathrm{~mm}$ has been transformed into an expanded hot liquid state while the melting front is seen moving outwards.

Figure 4 also shows the material physical state, but at $t=2.5 \mu \mathrm{s}$. It is seen that within the inner $2 \mathrm{~mm}$ radius, a

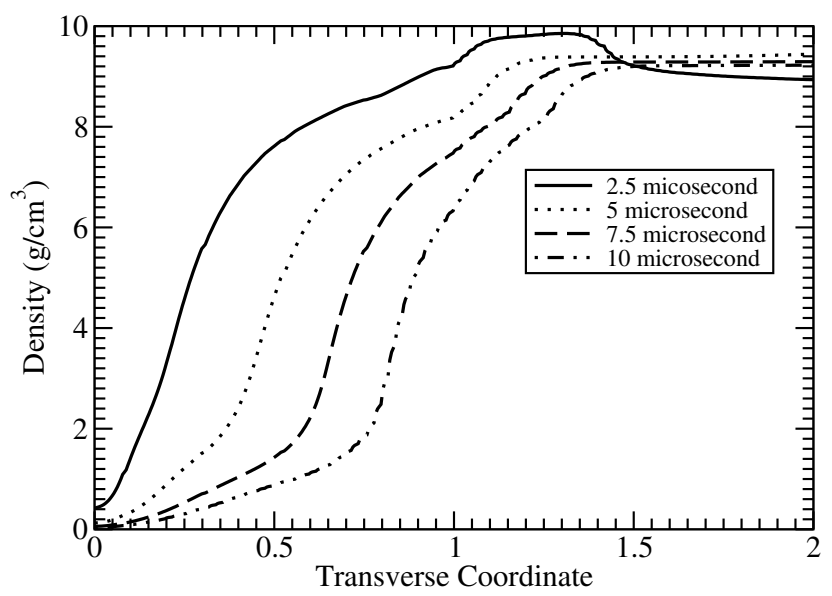

FIG. 6. Density vs transverse coordinate at different times at a longitudinal position of $16 \mathrm{~cm}$.

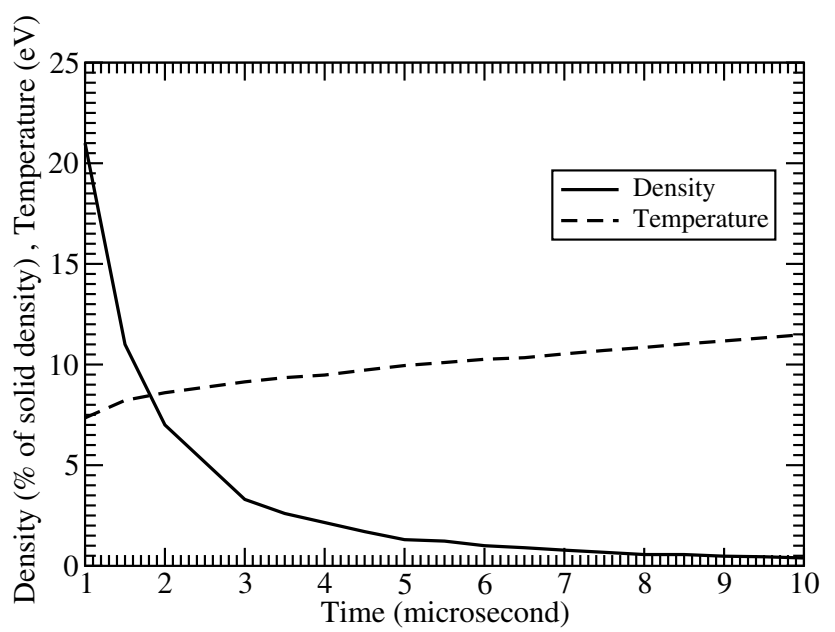

FIG. 7. Density and temperature at the target center vs time.

plasma is created and the plasma region is followed by a liquid region that extends up to a radius of $8 \mathrm{~mm}$.

In Figs. 5 and 6 we plot the temperature and density vs the transverse coordinate at different times, namely, 2.5, 5, 7.5 , and $10 \mu$ s respectively.

It is seen from Fig. 5 that the maximum temperature at the target axis at $t=2.5 \mu \mathrm{s}$ is about $9 \mathrm{eV}$ and by this time 100 bunches have deposited their energy in the target that leads to a specific energy deposition of the order of $200 \mathrm{~kJ} / \mathrm{g}$. The temperature increases as more energy is deposited by the subsequent bunches, but since the material expands rapidly, the temperature increase is not significant. Figure 6 shows that the density decreases with time as a result of the outgoing shock wave.

In Fig. 7 we plot the density and temperature at the target center vs time. In Figs. 8 and 9 we plot the plasma coupling parameter $\Gamma$ and the degree of ionization in a copper plasma as a function of temperature and density, respectively. Calculations of the plasma coupling parameter have

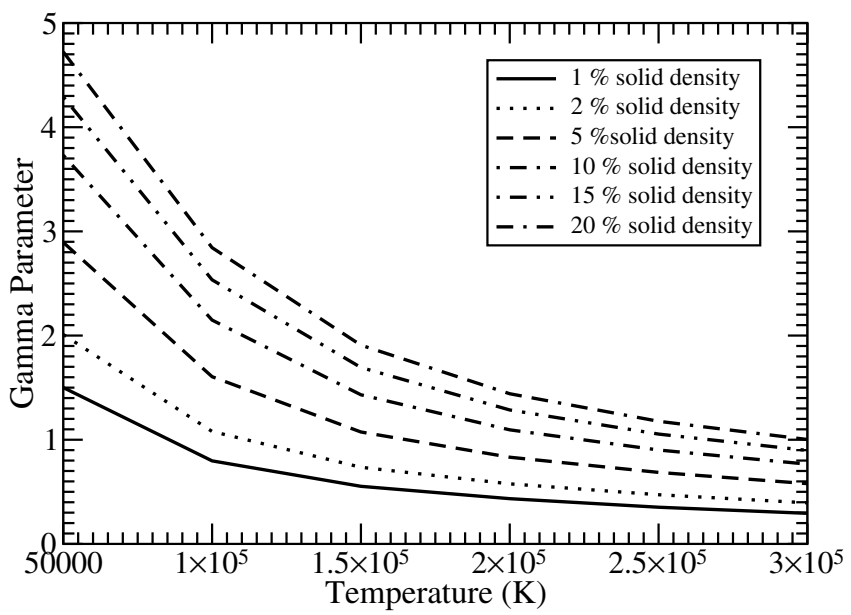

FIG. 8. Plasma coupling parameter $\Gamma$ as a function of temperature and density. 


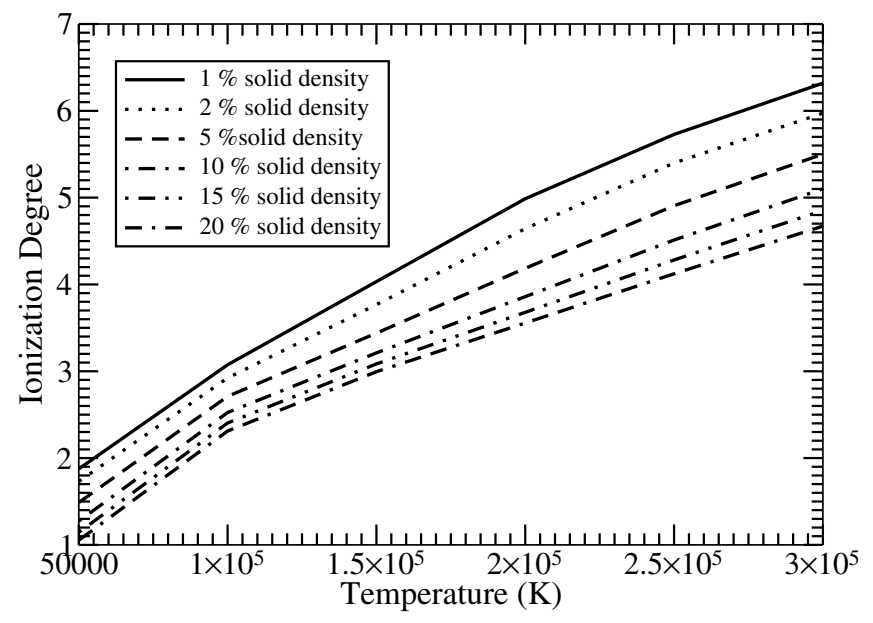

FIG. 9. Degree of ionization in the plasma as a function of temperature and density.

been performed using the code SAHA-IV [17-19], which is specially designed for calculations of thermodynamic properties of multicomponent plasma with strong interparticle interactions. The calculation procedure is based on chemical picture of the plasma [17,18]. Coulomb interaction of charged particles, short range repulsion of atoms and ions at close distances, degeneracy of free electrons, and stages of ionization up to 20 were taken into account. Further details can be found in [19].

A comparison between Fig. 7 and Fig. 8 shows that at the central part of the target, a strongly coupled plasma with a plasma parameter of the order of $2-5$ is created. Similarly a comparison between Fig. 7 and Fig. 9 shows that the degree of ionization in the plasma will be from 2 to 3 . An LHC beam will therefore be a very efficient tool to create states of an expanded hot liquid and strongly coupled plasmas with lifetimes of the order of $10 \mu \mathrm{s}$. We note that at $t=10 \mu \mathrm{s}$, only about 400 out of 2808 LHC bunches have been delivered. However, by this time the target density becomes extremely low, the rest of the bunches will encounter little mass and will therefore pass through this region of the target without any significant interaction and will penetrate deeper into the target. The time for investigation may therefore be limited to $10 \mu \mathrm{s}$, which is sufficiently long to carry out diagnostics.
Diagnostics of such samples of HED matter will be a very challenging problem. A complete set of diagnostic tools will be needed to measure the density, temperature, and pressure of the sample to fully determine the EOS. The simulations show a temperature of the order of $10 \mathrm{eV}$ that corresponds to a maximum of the Planck radiation of a wavelength of about $30 \mathrm{~nm}$, that will make pyrometry in the uv region a possibility to measure the temperature. Piezoelectric polymer stress gauges that are immersed in the target can be used to measure the pressure. The density can be measured using $\mathrm{x}$-ray backlighting and shadowgraphy technique as well as proton radiography.

[1] R. G. McQueen et al., in High Velocity Impact Phenomena, edited by R. Kinslow (Academic, New York, 1970), p. 293, appendices on p. 515.

[2] M. van Thiel, Lawrence Livermore Laboratory Report, Report No. UCRL-50108, 1977.

[3] LASL Shock Hugoniot Data, edited by S.P. Marsh (University of California, Berkeley, 1980).

[4] M. V. Zhernokletov et al., Experimental Data on Shock Compression and Adiabatic Expansion of Condensed Materials at High Energy Density (IPCPRAS, Chernogolovka, 1996) [in Russian].

[5] M. D. Knudson et al., Phys. Rev. Lett. 87, 225501 (2001).

[6] A. S. Vladimirov et al., Sov. Phys. JETP 39, 85 (1984).

[7] Th. Löwer et al., Phys. Rev. Lett. 72, 3186 (1994).

[8] R. Cauble et al., Phys. Rev. Lett. 70, 2102 (1993).

[9] M. Koenig et al., Phys. Rev. Lett. 74, 2260 (1995).

[10] D. Batani et al., Phys. Rev. B 61, 9287 (2000).

[11] D. H. H. Hoffmann et al., Phys. Plasmas 9, 3651 (2002).

[12] V. E. Fortov et al., Nucl. Sci. Eng. 123, 169 (1996).

[13] A. V. Bushman and V. E. Fortov, Sov. Tech. Rev. B Therm. Phys. 1, 219 (1987).

[14] C. Deutsch, Ann. Phys. (Paris) 11, 1 (1986).

[15] T. A. Mehlhorn, J. Appl. Phys. 52, 6522 (1981).

[16] A. Fasso et al., hep-ph/0306267.

[17] V. K. Gryaznov et al., in Thermophysical Properties of Working Media of Gase-Phase Nuclear Reactor, edited by V. M. Ievlev (Atomizdat, Moscow, 1980).

[18] W. Ebeling et al., Thermophysical Properties of Hot Dense Plasmas (Teubner, Stuttgart-Leipzig, 1991).

[19] V. K. Gryaznov et al., Zh. Eksp. Teor. Fiz. 114, 1242 (1998). 